\title{
BMJ Open Post-traumatic stress disorder psychological interventions in sub- Saharan Africa: protocol for a systematic review of the literature
}

\author{
Vuyokazi Ntlantsana (D) , ${ }^{1}$ Keneilwe Molebatsi (D) , , ${ }^{1,2}$ Sibongile Mashaphu, ${ }^{1}$ \\ Bonginkosi Chiliza (D) , ${ }^{1}$ Dickens Akena (D) ${ }^{3}$
}

To cite: Ntlantsana V,

Molebatsi K, Mashaphu S, et al. Post-traumatic stress disorder psychological interventions in sub-Saharan Africa: protocol for a systematic review of the literature. BMJ Open 2022;12:e052903. doi:10.1136/ bmjopen-2021-052903

- Prepublication history and additional supplemental material for this paper are available online. To view these files, please visit the journal online (http://dx.doi.org/10.1136/ bmjopen-2021-052903).

Received 07 May 2021 Accepted 01 February 2022

Check for updates

(c) Author(s) (or their employer(s)) 2022. Re-use permitted under CC BY-NC. No commercial re-use. See rights and permissions. Published by BMJ.

${ }^{1}$ Departments of Psychiatry, University of KwaZulu-Natal, Durban, South Africa ${ }^{2}$ Department of Psychiatry, University of Botswana, Gaborone, Botswana ${ }^{3}$ Department of Psychiatry, Makerere University, Kampala, Uganda

Correspondence to Dr Vuyokazi Ntlantsana; Ntlantsanav@ukzn.ac.za

\section{ABSTRACT}

Introduction Untreated post-traumatic stress disorder (PTSD) results in considerable morbidity and higher risk of mortality. However, little is being done to treat PTSD in sub-Saharan Africa (SSA) settings where rates of PTSD are likely to be elevated due to mass exposure of the population to traumatic events. This systematic review aims to summarise available evidence on the efficacy of psychological therapies for PTSD in SSA.

Methods and analysis A systematic search of the literature will be conducted in four electronic databases: PubMed, Psychlnfo, EMBASE and Cochrane Register of Controlled Trials using keywords and synonyms related to 'PTSD', 'psychological therapies' and 'SSA'. Studies will be included if they were conducted in SSA and used a randomised controlled trial design. Two researchers will screen the studies for eligibility to be included, and data on intervention types, population, comorbidities, comparison group intervention and PTSD outcomes will be extracted from those included. Risk of bias will be assessed using Cochrane risk-of-bias tool for randomised trials (version 2). Narrative synthesis of data will be conducted and pooled effect estimates calculated.

Ethics and dissemination Ethical approval is not required as this is a protocol of a systematic review of available data. Findings will be disseminated to the scientific community through peer-reviewed publications and presentation at conferences.

PROSPERO registration CRD42020181638.

\section{INTRODUCTION}

Post-traumatic stress disorder (PTSD) is a debilitating mental illness and occurs at a prevalence of $3.9 \%$ and $5.6 \%$ in general and trauma exposed populations, respectively. A higher prevalence of $15.5 \%$ has been reported in conflict affected populations and up to $24 \%$ in war survivors. ${ }^{2}{ }^{3}$ During the COVID-19 pandemic, a PTSD prevalence of $44.5 \%$ and $55.1 \%$ was reported in certain groups, such as healthcare workers in South Africa and Kenya, respectively. ${ }^{45}$ Untreated, PTSD results in considerable morbidity (increase in years lived with disability), higher
Strengths and limitations of this study

$\Rightarrow$ This review is an expansion of the existing literature on post-traumatic stress disorder (PTSD) interventions in sub-Saharan Africa (SSA).

$\Rightarrow$ Included studies may be heterogenous, limiting the pooling of data and ability to generalise findings.

$\Rightarrow$ Considering the burden of PTSD in SSA, findings from this systematic review will be important to inform future PTSD interventions for optimum outcomes.

$\Rightarrow$ The review will include a comparator group, thereby adding to the strength of the evidence.

risk of developing cardiovascular disease, premature mortality due to an accelerated ageing process and a higher risk of suicide. ${ }^{6-9}$ Owing to the high burden of PTSD and the associated negative adversities, PTSD should be identified and treated timeously. ${ }^{10}{ }^{11}$ However, little has been done to identify and treat PTSD in low resource sub-Saharan African (SSA) settings where multiple civil and tribal wars, social strife and natural disasters such as floods and landslides, have led to mass exposure of the population to traumatic events. ${ }^{12-14}$

Several reasons could explain the poor identification and treatment of PTSD in SSA, including that mental health services account for low expenditure of the total budget allocation towards healthcare. In South Africa, for example, only $5 \%$ of the national health budget goes towards mental health expenditure, of which $86 \%$ is spent on inpatient care and only $8 \%$ on primary healthcare. ${ }^{1516}$ This accounts for lower access to mental healthcare for most of the population, who can only access community level services. Some patients have spiritual and cultural beliefs about mental health conditions, that may be different from conventional psychiatry 
diagnosis. These patients may find meaning consulting traditional or other healers. ${ }^{17}$ Aggarwal $e t$ al ${ }^{18}$ in their study including patients, clinicians and administrators found that low levels of cultural competency and the inability to provide culturally appropriate healthcare services, widens the therapeutic alliance gap and results in mistrust of services provided by conventional healthcare providers. ${ }^{18}$ Some individuals with PTSD do not volunteer information to healthcare workers or seek help due to the stigmatising attitudes of healthcare workers, community members and self-stigma about mental illness. ${ }^{119-23}$ Additionally, there is a general lack of knowledge that PTSD is a medical condition, creating a further barrier to seeking care. ${ }^{1124}$ Comorbidities such as depression, anxiety and substance use disorders may negatively impact on the clinician's ability to diagnose PTSD. ${ }^{24-26}$ Furthermore, limited access to mental healthcare, and severe shortage of trained or professional mental healthcare staff hampers the identification and treatment of PTSD. An average of 1.4 mental health workers are available per 100000 persons in Africa compared with the global average of 9 per $100000 .^{152728}$ The provision of specialised community-based services, collaborative care interventions, task-shifting and task sharing approaches are some of the strategies implemented to strengthen mental healthcare provision in low resource settings. ${ }^{29} 30$ Specialised community-based services entail formal links between primary and specialist care, where professional mental healthcare providers run services that are located at community level. ${ }^{29}$ In collaborative care interventions, mental healthcare provision is provided in a structured way between the various types of providers. ${ }^{29} 30$ Task shifting and task sharing is the provision of mental healthcare services by individuals who have had no professional health training. ${ }^{31}$ In task shifting, these services can be provided by individuals with less training, for example, nurses or lay health workers.

Psychological therapy, also called talk therapy is an effective psychological intervention used for psychological, behavioural and somatic symptoms. ${ }^{32} 33$ and can be delivered in groups or individually. ${ }^{34}$ A number of efficacious PTSD interventions have been cited in the literature and these include cognitive based interventions which may be trauma or non-trauma focused, eye movement desensitisation and reprocessing (EMDR) and other therapies including psychodynamic therapy, hypnotherapy, supportive counselling and present-centred counselling. ${ }^{34}$

A number of studies that assessed the efficacy of psychological interventions for PTSD have been conducted in SSA ${ }^{35-37}$ Some interventions have been found to be efficacious, but others have had negative findings with methodological flaws reported as the reasons for some of the negative findings.

In a Rwandan cohort of genocide survivors, Connolly and Sakai ${ }^{38}$ described the sustained reduction of PTSD symptoms with the trauma exposure-based intervention, thought field therapy. There was a 32\% $(\mathrm{p}<0.01)$ reduction in PTSD symptoms in the treatment group, while the waitlist group had a symptom reduction of $12.1 \%$ ( $>>0.05)$. Improvement in the treatment group was sustained at the 2-year follow-up assessment. In a Mozambican community sample of individuals with PTSD, Igreja et $a l^{36}$ found the testimony intervention procedure which entailed participants giving a verbal account of the most traumatic event to be effective in reducing PTSD symptoms. The efficacy of the intervention was sustained for at least 11 months, particularly among the female participants. There was no significant difference between cases and controls in the immediate (2 months) post-intervention phase. Traumafocused cognitive behavioural therapy was found to be superior to treatment as usual in a randomised controlled trial (RCT) in a cohort of orphaned and vulnerable children with trauma related and PTSD symptoms in Lusaka, Zambia. ${ }^{37}$ The intervention was administered by trained lay counsellors, but post-intervention assessments were only conducted once, 1 month after the intervention, and it was therefore unclear if the effects of the intervention were sustained over time.

Despite the availability of these interventions in SSA, healthcare workers continue to be faced with a dilemma of which intervention to use, in part due to the differences in populations in which the interventions were administered as well as the type, methods and duration of administration, with some being brief and others long.

Due to cultural diversity within the SSA region, as well as the type and extent of trauma exposure between the countries across the region, we hypothesise that the efficacy of psychological interventions may differ across SSA. Therefore, the aim of this review is to summarise the available evidence about the efficacy of psychological therapies for PTSD in sub-Saharan Africa, to determine the types of interventions found to be effective and to describe the contexts in which these interventions have been effective. This will help guide clinicians faced with the dilemma of selecting appropriate interventions for their setting.

\section{METHODS AND ANALYSIS}

The systematic review is registered with Prospective Register of Systematic Reviews. Article selection will be reported according to the Preferred Reporting Items for Systematic Reviews and Meta-Analysis guidelines in figure $1 .{ }^{39}$

\section{Literature search}

A literature search of articles published between 1980 and November 2021 will be conducted on PubMed, PsychInfo, EMBASE and Cochrane Register of Controlled Trials electronic databases. The review will include studies from 1980 as this is the first time PTSD was described. ${ }^{40} \mathrm{~A}$ search for grey literature will be conducted by searching for abstracts of conferences for relevant studies. Reference lists of included articles will be screened for relevant articles. The ClinicalTrials.gov website and International Clinical Trials Registry Platform will also be searched for 


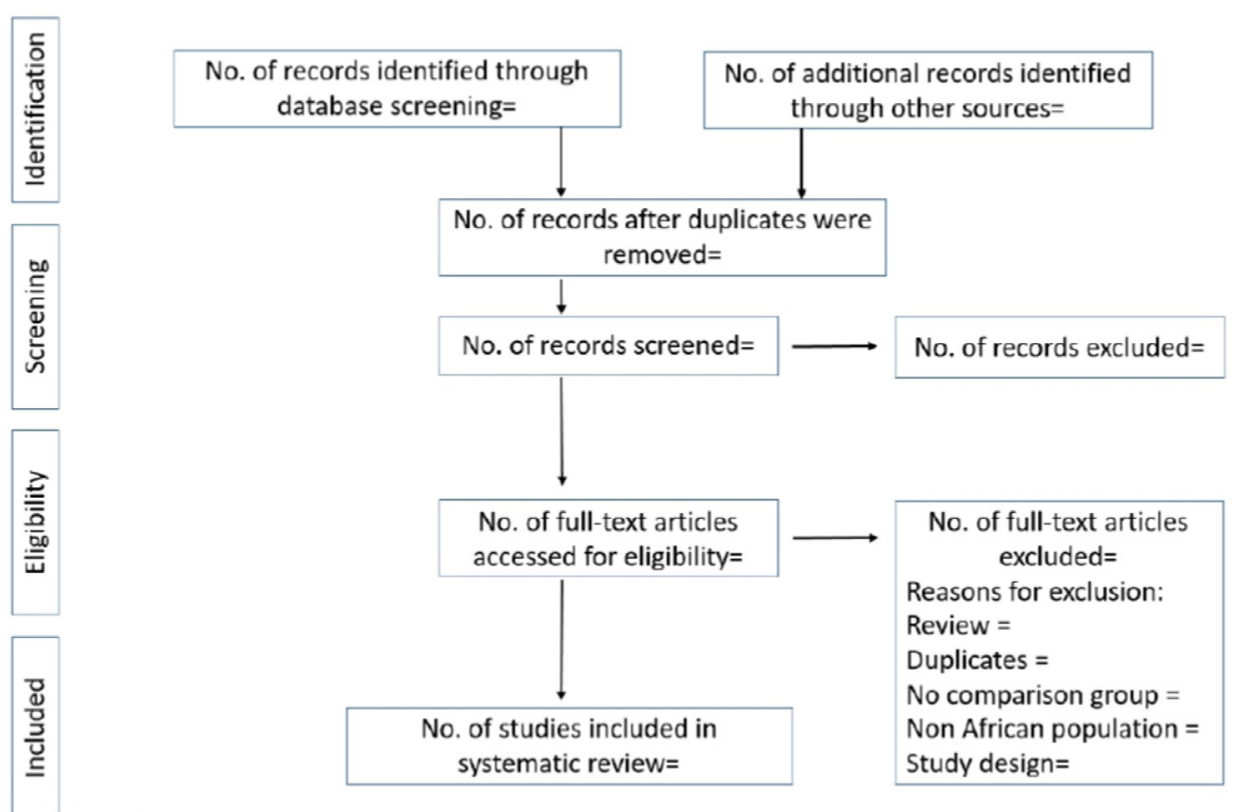

Figure 1 PRISMA flow diagram (adapted from Moher et $\mathrm{a} /{ }^{39}$ ). PRISMA, Preferred Reporting Items for Systematic Reviews and Meta-Analysis.

relevant studies. A librarian trained in systematic reviews will be enlisted when conducting the literature search.

\section{Study eligibility criteria}

The review will include all types of RCTs of psychological interventions for PTSD in populations in SSA. Eligible studies in all languages will be included and professional academic language translating services available at the institution of one of the co-authors (DA) will be solicited.

\section{Population}

Studies will be included if they have participants aged 18 years and above and diagnosed with PTSD as a primary diagnosis according to Diagnostic Statistical Manual of Mental Disorders (DSM) or International Classification of Diseases criteria. PTSD diagnosis will be considered if structured clinical interviews are used to make the diagnosis. This will include the use of PTSD diagnostic tools such as the Clinician-Administered PTSD Scale, PTSD Symptom Scale Interview, Mini-International Neuropsychiatric Interview and the Structured Clinical Interview for DSM. ${ }^{41-43}$

\section{Intervention}

Studies on any psychological group or individually administered interventions found will be included in the review. These will include, but not be limited to, traumabased, cognitive-based EMDR and relaxation-based interventions.

\section{Comparison}

Studies with comparator groups, such as treatment as usual, wait-listed groups or groups that have received other psychological therapies, will be included.

\section{Outcome}

The primary outcome measure will be a reduction in PTSD symptoms (measured by a valid instrument), based on measures of the scores being used. Secondary outcome measures will include dropout rates, other psychiatric comorbidities (eg, associated depression or substance use disorders), level of functioning, quality of life, as well as the level of training of individuals administering the intervention.

\section{Comorbidities}

Participants with other comorbidities will be included in the study, as long as the interventions in question target PTSD symptomatology and not comorbidities.

\section{Timing of outcome measures}

Outcome measures will be assessed at the period immediately after intervention, at 1-3 months, 4-6 months, 6-12 months and over 12 months after the intervention.

\section{Data collection}

Study selection

A pilot database search has been conducted and the results can be found in online supplemental additional file 1. Articles obtained from the electronic search conducted by the lead author (VN) and the Librarian will be exported to an EndNote V.X9 library. Duplicates will be removed from the library and it will be shared with the second reviewer (KM). The two reviewers will independently read all abstracts of articles identified during the literature search, to identify abstracts potentially meeting inclusion criteria. Thereafter, full-text articles of included abstracts will be screened for final inclusion in the study. Articles where reviewer discrepancies are found 
will be re-evaluated and a third reviewer (SM) will help reach a consensus. Cohen's kappa to assess for inter-rater agreement at abstract and full article inclusion stage will be reported.

\section{Data extraction}

Data from included studies will be extracted in duplicate, independently, by the reviewers onto Review Manager 5 Software (RevMan5). The extracted data will include participant demographic information, type of population (immigrants vs general population), urban versus rural, type of trauma, randomisation procedure, psychological intervention type and duration, information on outcomes and sources of funding. Authors will be contacted for missing information.

\section{Risk of bias assessment}

Risk of publication bias assessment based on PTSD symptoms will be conducted using a funnel plot, where asymmetry of the plot may be indicative of bias. Individual studies will be assessed for selection bias by assessing how intervention allocation was conducted, information bias by assessing if outcome assessors were blinded, and if analysis was biased by assessing if intention to treat analysis was used. Version 2 of the Cochrane risk-of-bias for randomised trials tool will be used to evaluate risk of bias in the included studies. ${ }^{44}$

\section{Data analysis}

Review Manager V.5 software will be used for pooled effect estimates where possible. Data will be pooled where suitable and analysed for summary effects. Comparisons will be made between outcome measures (dependent variables) in the group that did and did not receive the intervention (independent variable). For dichotomous data such as PTSD present or not present at the end of the intervention, or response or no response to treatment, effect sizes will be calculated as the ORs. For continuous data such as PTSD scores, level of functioning and quality of life scores, mean differences between the intervention and control or waitlisted groups will be calculated. Standardised mean differences will be calculated where different measures or tools are used to assess outcomes. For crossover trials, data will be extracted from the first treatment period. Sub-analysis comparing the different types of interventions, PTSD severity, comorbid psychiatric conditions versus no comorbid condition will be conducted. Sub-analysis of how the presence of the different types of comorbidities, for example, depressive versus substance use comorbidities, are associated with response to the intervention, will also be conducted. Comparisons will also be made by the type of interventionist, whether healthcare professional, community professional or lay health worker. Random effects metaanalysis and inverse variance weighting methods will be used to account for expected heterogeneity of included studies. A narrative description of the studies and findings will also be undertaken. Interventions with similar core components or population groups will be pooled together to make informative clinical inferences. Sensitivity analysis will be conducted by conducting statistical tests with and without studies that may not meet the minimum criteria for risk of bias, and tests with and without grey literature. If findings remain consistent, the robustness of the findings will be assured, but if findings change with exclusion of certain studies, then risk of reporting bias will be considered. Overall, the level of evidence for this review will be assessed using the Grading of Recommendations, Assessment, Development and Evaluation system. ${ }^{45}$ The a priori level of evidence is high as the review will include only RCTs, but the ultimate quality will depend on other findings such as effect size and risk of bias assessment.

\section{Public and patient involvement}

Public and patient involvement has not been included in the design of this systematic review as the review will use information readily available in the public domain.

\section{ETHICS AND DISSEMINATION}

This is a systematic review of publicly available data and ethical approval is not required. Findings will be published in a peer-reviewed journal and presented to scientific audiences at local and international conferences.

Contributors VN, KM, SM, BC and DA were involved with the concept, design and data analysis plan of the review. VN was lead author and all authors contributed to the write-up and editing of manuscript and approval of the final version of the manuscript. DA is the guarantor of the manuscript.

Funding The publication was financially supported by the Schizophrenia Research Society (SIRS), and by the University of KwaZulu-Natal Research Innovation and Sequencing Platform (KRISP).

Competing interests None declared.

Patient consent for publication Not applicable.

Provenance and peer review Not commissioned; externally peer reviewed.

Supplemental material This content has been supplied by the author(s). It has not been vetted by BMJ Publishing Group Limited (BMJ) and may not have been peer-reviewed. Any opinions or recommendations discussed are solely those of the author(s) and are not endorsed by BMJ. BMJ disclaims all liability and responsibility arising from any reliance placed on the content. Where the content includes any translated material, BMJ does not warrant the accuracy and reliability of the translations (including but not limited to local regulations, clinical guidelines, terminology, drug names and drug dosages), and is not responsible for any error and/or omissions arising from translation and adaptation or otherwise.

Open access This is an open access article distributed in accordance with the Creative Commons Attribution Non Commercial (CC BY-NC 4.0) license, which permits others to distribute, remix, adapt, build upon this work non-commercially, and license their derivative works on different terms, provided the original work is properly cited, appropriate credit is given, any changes made indicated, and the use is non-commercial. See: http://creativecommons.org/licenses/by-nc/4.0/.

\section{ORCID iDs}

Vuyokazi Ntlantsana http://orcid.org/0000-0002-5882-100X Keneilwe Molebatsi http://orcid.org/0000-0003-4741-3278

Bonginkosi Chiliza http://orcid.org/0000-0001-5417-5920

Dickens Akena http://orcid.org/0000-0002-8886-4553 


\section{REFERENCES}

1 Koenen KC, Ratanatharathorn A, Ng L, et al. Posttraumatic stress disorder in the world mental health surveys. Psychol Med 2017;47:2260-74.

2 Hoppen TH, Morina N. The prevalence of PTSD and major depression in the global population of adult war survivors: a meta-analytically informed estimate in absolute numbers. Eur $\mathrm{J}$ Psychotraumatol 2019;10:1578637.

3 Charlson F, van Ommeren M, Flaxman A, et al. New WHO prevalence estimates of mental disorders in conflict settings: a systematic review and meta-analysis. Lancet 2019;394:240-8.

4 Engelbrecht MC, Heunis JC, Kigozi NG. Post-Traumatic stress and coping strategies of South African nurses during the second wave of the COVID-19 pandemic. Int J Environ Res Public Health 2021;18:7919.

5 Asnakew S, Legas G, Muche Liyeh T, et al. Prevalence of posttraumatic stress disorder on health professionals in the era of COVID-19 pandemic, Northwest Ethiopia, 2020: a multi-centered cross-sectional study. PLoS One 2021;16:e0255340.

6 Bach SdeL, Molina MAL, Jansen K, et al. Suicide risk and childhood trauma in individuals diagnosed with posttraumatic stress disorder. Trends Psychiatry Psychother 2018;40:253-7.

7 Brown LA, Contractor A, Benhamou K. Posttraumatic stress disorder clusters and suicidal ideation. Psychiatry Res 2018;270:238-45.

8 Gradus JL. Posttraumatic stress disorder and death from suicide. Curr Psychiatry Rep 2018;20:98.

9 Lohr JB, Palmer BW, Eidt CA, et al. Is post-traumatic stress disorder associated with premature senescence? A review of the literature. Am J Geriatr Psychiatry 2015;23:709-25.

10 Watkins LE, Sprang KR, Rothbaum BO. Treating PTSD: a review of evidence-based psychotherapy interventions. Front Behav Neurosci 2018;12:258

11 Greenberg N, Brooks S, Dunn R. Latest developments in posttraumatic stress disorder: diagnosis and treatment. Br Med Bull 2015;114:147-55.

12 Smigelsky MA, Aten JD, Gerberich S, et al. Trauma in sub-Saharan Africa: review of cost, estimation methods, and interventions. Int J Emerg Ment Health 2014;16:354-65.

13 Alfa-Wali M, Sritharan K, Mehes M, et al. Terrorism-related trauma in Africa, an increasing problem. J Epidemiol Glob Health 2015:5:201-3.

14 Chol C, Negin J, Garcia-Basteiro A, et al. Health system reforms in five sub-Saharan African countries that experienced major armed conflicts (wars) during 1990-2015: a literature review. Glob Health Action 2018;11:1517931.

15 Sankoh O, Sevalie S, Weston M. Mental health in Africa. Lancet Glob Health 2018;6:e954-5.

16 Docrat S, Besada D, Cleary S, et al. Mental health system costs, resources and constraints in South Africa: a national survey. Health Policy Plan 2019;34:706-19.

17 Nortje G, Oladeji B, Gureje O, et al. Effectiveness of traditional healers in treating mental disorders: a systematic review. Lancet Psychiatry 2016;3:154-70.

18 Aggarwal NK, Cedeño K, Guarnaccia P, et al. The meanings of cultural competence in mental health: an exploratory focus group study with patients, clinicians, and administrators. Springerplus 2016;5:384.

19 Said M, Boardman G, Kidd S. Barriers to accessing mental health services in Somali-Australian women: a qualitative study. Int $\mathrm{J}$ Ment Health Nurs 2021;30:931-8.

20 Tesfaye Y, Agenagnew L, Terefe Tucho G, et al. Attitude and helpseeking behavior of the community towards mental health problems. PLoS One 2020;15:e0242160.

21 Zhang Y, Augusto O, Ásbjörnsdóttir K, et al. Geographic distribution and determinants of mental health stigma in central Mozambique. Soc Psychiatry Psychiatr Epidemiol 2019;54:1391-410.
22 Rugema L, Persson M, Mogren I, et al. A qualitative study of healthcare professionals' perceptions of men and women's mental healthcare seeking in Rwanda. J Community Psychol 2020;48:891-903.

23 Ubaka CM, Chikezie CM, Amorha KC, et al. Health professionals' stigma towards the psychiatric ill in Nigeria. Ethiop $\mathrm{J}$ Health Sci 2018;28:483-94.

24 Sareen J. Posttraumatic stress disorder in adults: impact, comorbidity, risk factors, and treatment. Can J Psychiatry 2014:59:460-7.

25 Van Zyl M, Oosthuizen PP, Seedat S. Post traumatic stress disorder: undiagnosed cases in a tertiary inpatient setting. Afr J Psychiatry 2008;11:119-22.

26 Klaric M, Lovric S, Kresic Coric M, et al. Psychiatric comorbidity and PTSD-related health problems in war veterans: cross-sectional study. The European Journal of Psychiatry 2017;31:151-7.

27 World Health Organisation,. mhGAP intervention guide for mental, neurological and substance use disorders in non-specialized health settings. WHO Geneva, 2010.

28 Semrau M, Evans-Lacko S, Alem A, et al. Strengthening mental health systems in low- and middle-income countries: the Emerald programme. BMC Med 2015;13:79.

29 Mapanga W, Casteleijn D, Ramiah C, et al. Strategies to strengthen the provision of mental health care at the primary care setting: an evidence MAP. PLoS One 2019;14:e0222162.

30 van Ginneken N, Chin WY, Lim YC, et al. Primary-level worker interventions for the care of people living with mental disorders and distress in low- and middle-income countries. Cochrane Database Syst Rev 2021;8:CD009149.

31 Galvin M, Byansi W. A systematic review of task shifting for mental health in sub-Saharan Africa. Int J Ment Health 2020;49:336-60.

32 Locher C, Meier S, Gaab J. Psychotherapy: a world of meanings. Front Psychol 2019;10:460.

33 Parekh R, Givon L, eds. What is Psychotherapy? APA, 2019

34 Bisson Jl. Psychological therapies for chronic post-traumatic stress disorder (PTSD) in adults. Cochrane database Syst Rev 2015;12.

35 Connolly S, Sakai C. Brief trauma intervention with Rwandan genocide-survivors using thought field therapy. Int J Emerg Ment Health 2011;13:161.

36 Igreja V, Kleijn WC, Schreuder BJN, et al. Testimony method to ameliorate post-traumatic stress symptoms. community-based intervention study with Mozambican civil war survivors. $\mathrm{Br} \mathrm{J}$ Psychiatry 2004;184:251-7.

37 Murray LK, Skavenski S, Kane JC, et al. Effectiveness of traumafocused cognitive behavioral therapy among trauma-affected children in Lusaka, Zambia: a randomized clinical trial. JAMA Pediatr 2015;169:761-9.

38 Connolly S, Sakai C. Brief trauma intervention with Rwandan genocide-survivors using thought field therapy. Int J Emerg Ment Health 2011;13): :161-72.

39 Moher D, Liberati A, Tetzlaff J, et al. Preferred reporting items for systematic reviews and meta-analyses: the PRISMA statement. Ann Intern Med 2009;151:264-9.

40 Friedman MJ. PTSD history and overview, 2018.

41 Weathers FW, Bovin MJ, Lee DJ, et al. The Clinician-Administered PTSD scale for DSM-5 (CAPS-5): development and initial psychometric evaluation in military veterans. Psychol Assess 2018;30:383-95.

42 Foa EB, McLean CP, Zang Y, et al. Psychometric properties of the posttraumatic stress disorder symptom scale interview for DSM-5 (PSSI-5). Psychol Assess 2016;28:1159-65.

43 First Met al. Structured clinical interview for the DSM-IV axis I disorders (SCID PTSD module. National Center for PTSD, 1996.

44 Sterne JAC, Savović J, Page MJ, et al. RoB 2: a revised tool for assessing risk of bias in randomised trials. BMJ 2019;366:14898.

45 Aguayo-Albasini JL, Flores-Pastor B, Soria-Aledo V. [GRADE system: classification of quality of evidence and strength of recommendation]. Cir Esp 2014;92:82-8. 
Correction: Post-traumatic stress disorder psychological

interventions in sub-Saharan Africa: protocol for a

systematic review of the literature

Ntlantsana V, Molebatsi K, Mashaphu S, et al. Post-traumatic stress disorder psychological interventions in sub-Saharan Africa: protocol for a systematic review of the literature. BMJ Open 2022;12:e052903. doi: 10.1136/bmjopen-2021-052903.

The authors want to alert the readers on the updated Funding statement.

Funding: The publication was financially supported by the Schizophrenia Research Society (SIRS), and by the University of KwaZulu-Natal Research Innovation and Sequencing Platform (KRISP).

Open access This is an open access article distributed in accordance with the Creative Commons Attribution Non Commercial (CC BY-NC 4.0) license, which permits others to distribute, remix, adapt, build upon this work non-commercially, and license their derivative works on different terms, provided the original work is properly cited, appropriate credit is given, any changes made indicated, and the use is non-commercial. See: http://creativecommons.org/licenses/by-nc/4.0/.

( ) Author(s) (or their employer(s)) 2022. Re-use permitted under CC BY-NC. No commercial re-use. See rights and permissions. Published by BMJ.

BMJ Open 2022;12:e052903corr1. doi:10.1136/bmjopen-2021-052903corr1

Check for updates 